\title{
The CantiClever: A Dedicated Probe for Magnetic Force Microscopy
}

\author{
Arnout van den Bos, Iwan Heskamp, Martin Siekman, Leon Abelmann, and Cock Lodder
}

\begin{abstract}
We present a new cantilever for magnetic-force microscopy (MFM), the CantiClever, which is not derived from atomic-force microscopy (AFM) probes but optimized for MFM. Our design integrates the cantilever and the magnetic tip in a single manufacturing process with the use of silicon micromachining techniques, which allows for batch fabrication of the probes. This manufacturing process enables precise control on all dimensions of the magnetic tip, resulting in a very thin magnetic element with a very high aspect ratio. Using the CantiClever, magnetic features down to $30 \mathrm{~nm}$ could be observed in a CAMST reference sample.
\end{abstract}

Index Terms-Cantilevers, integration, magnetic force microscopy (MFM), magnetic tips.

\section{INTRODUCTION}

W ITH magnetic force microscopy (MFM) a high-resolution image of the stray field of a magnetic sample can be obtained. A typical resolution of $50 \mathrm{~nm}$ can be achieved with very little sample preparation [1]. This makes MFM a very useful tool for the study and characterization of magnetic materials for high-density magnetic recording. As the areal density of magnetic recording systems increases very rapidly, the current resolution of MFM needs to be improved in order to remain useful as a measurement tool [2]. The resolution of MFM is determined by a combination of tip geometry and measurement noise. The thermal vibration of the cantilever currently determines the measurement noise levels in modern instruments; this thermal noise may be reduced by using cantilevers with low spring constants, high resonant frequencies, and high quality of resonance. This imposes a great challenge on the design of dynamic mode control electronics. However, at the moment it appears that the resolution of MFM is limited by the geometry of the magnetic tip. The dimensions as well as the shape of the magnetic tip have a big influence on the resolution. The ideal tip shape would be that of an elongated bar with a flat end, as depicted in Fig. 1 [3].

An elongated shape induces a strong shape anisotropy, which increases the stability of the magnetic tip against remagnetization by the sample. A long magnetic element also behaves as a monopole when imaging small magnetic features, which increases the signal output. Moreover, for thick films, a long tip also improves the output at very long wavelengths. The flat tip-end causes most of the magnetic charges to be confined as close as possible to the sample. This results in an improvement

Manuscript received February 14, 2002; revised May 28, 2002.

The authors are with the Systems and Materials for Information Storage Group, MESA ${ }^{+}$Research Institute, University of Twente, 7500 AE Enschede, The Netherlands (e-mail: a.g.vandenbos@el.utwente.nl).

Digital Object Identifier 10.1109/TMAG.2002.803585.

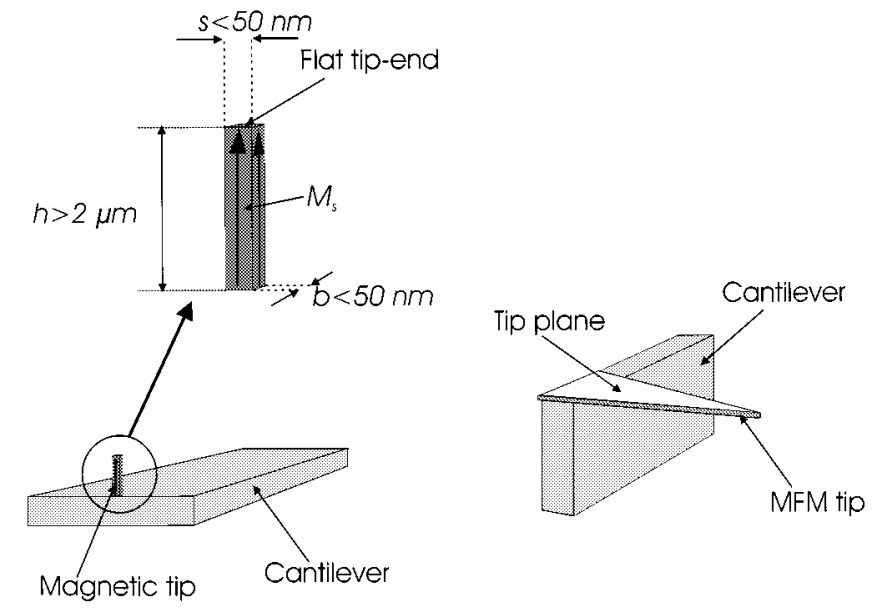

Fig. 1. Left: ideal tip shape for a MFM tip. Right: cantilever with the tip plane.

in resolution as compared to point sharp tips, which suffer from having a large proportion of their magnetic charges at a greater distance from the sample [4]. Commercially available MFM tips consist of an atomic-force microscopy (AFM) tip, coated with a thin layer of magnetic material, usually cobalt alloys [5]. The pyramidal shape of these tips does not bear much resemblance to the ideal tip shape mentioned above. A better approximation to this ideal shape is obtained with electron-beam-induced deposited (EBID) carbon needles [6]-[8], coated on one side with a thin magnetic layer. However, the EBID carbon needle has a shape similar to that of a cone. When coated with magnetic material, this causes not all the magnetic charges to be confined to the end of the tip, making the tip sensitive to stray fields from a larger area. Another major drawback of the EBID is their serial manufacturing process. Therefore, making one tip takes a considerable amount of time and, even worse, the tip shape is not exactly reproducible. Another method to make tips specially suited for MFM measurement is used by Phillips [9], who deposits magnetic material onto an AFM cantilever and uses focused ion-beam equipment to remove unwanted areas of the magnetic coating. With this serial production method $8-\mu \mathrm{m}-\mathrm{long}$ and 50-nm-wide needles could be realized. The new design presented in this paper results in an MFM tip with a nearly perfect shape that is produced in a highly reproducible batch manufacturing process.

\section{DESIGN}

A magnetic tip that is suitable for high-resolution MFM should have lateral dimensions in the nanometer regime. One would like these dimensions to be controllable and variable to 


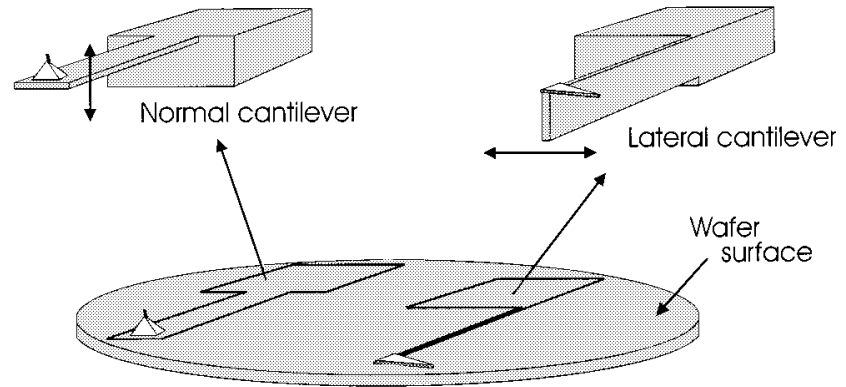

Fig. 2. Tip plane on the cantilever for both cantilever orientations. Left: conventional approach with perpendicularly vibrating cantilever; right: new approach with laterally vibrating cantilever.

be able to customize the MFM tip for different types of measurements or samples. The CantiClever design accomplishes this by defining both lateral dimensions by thin film deposition techniques. The magnetic tip is made by deposition of magnetic material on the side of a free-hanging, very thin layer called the tip plane. The width and thickness of the magnetic tip are defined by the thickness of the tip plane and the magnetic layer, respectively. The length of the tip is defined using photolithography. A schematic drawing of the structure is shown in Fig. 1.

Such a structure is very difficult to realize when using the conventional fabrication technique in which the cantilevers are situated such that the oscillation direction is perpendicular to the surface of the substrate. Using this approach, the very thin tip plane needs to be fabricated as a free-standing layer perpendicular to the substrate surface. To avoid this, the free-hanging tip plane is made with a completely new approach. During fabrication, the cantilevers are tilted $90^{\circ}$ compared to the conventional cantilevers, creating a laterally oscillating cantilever with its oscillation direction parallel to the substrate surface, as shown in Fig. 2.

This approach makes the fabrication of the cantilever more difficult compared to conventional cantilevers, but at the same time enables precise control over the cantilever resonance frequency which is given by [10]

$$
f_{0}=\frac{1}{2 \pi} C_{0}^{2} \frac{t}{l^{2}} \sqrt{\frac{E_{\mathrm{Si}}}{12 \rho_{\mathrm{Si}}}}
$$

where $C_{0}$ is the eigenvalue of the system corresponding to its fundamental frequency, having a value of $1.875, t$ the thickness of the cantilever, $l$ its length, $E_{\mathrm{Si}}$ the Young's modulus of silicon $(150 \mathrm{GPa})$ and $\rho_{\mathrm{Si}}$ the density of silicon $\left(2330 \mathrm{~kg} / \mathrm{m}^{3}\right)$. In contrast to the fabrication method used for conventional cantilevers, this approach allows both the cantilever length as well as the thickness to be controlled and varied lithographically, since both dimensions are defined parallel to the substrate surface. A single substrate can carry a large number of cantilevers with different resonance frequencies, suitable for different applications. Furthermore, standard deposition and etching techniques can be used to define the tip plane, as it is also oriented parallel to the substrate surface. The result is a reproducible manufacturing process that incorporates both the cantilever and the magnetic tip and allows for batch fabrication of the probes.

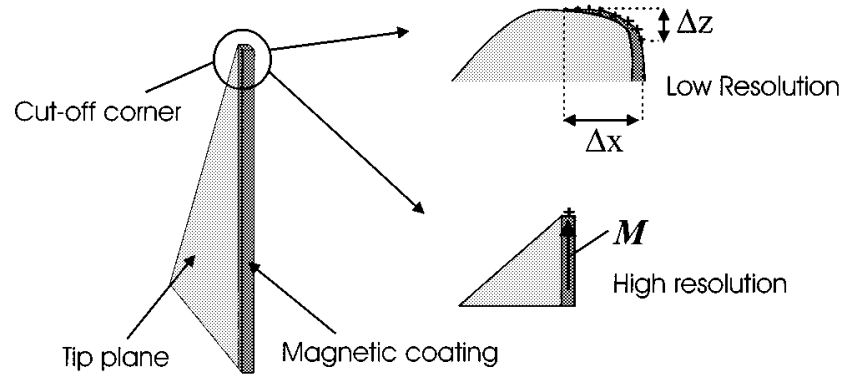

Fig. 3. Influence of the cutoff corner of the tip plane on the imaging resolution.

Two factors play an important role in realizing a high-resolution MFM tip with the ideal tip shape, as previously described. First, the free-standing tip plane should be very thin, as this defines the width of the tip, but also strong enough for contact imaging. At the same time, the tip plane material should have low residual stress to prevent bending after release. Second, the tip plane should have a well-defined and very sharp cutoff corner as illustrated in Fig. 3 and the etching process should be directional to ensure that the tip end remains flat.

\section{FABRICATION}

The cantilevers are fabricated from (110) silicon wafers using $\mathrm{KOH}$ wet anisotropic etching. The (110) oriented silicon wafers have a $(-1,1,1)$ and a $(1,-1,1)$ plane perpendicular to the wafer surface. The etch rate of the family of $\{111\}$ planes in $\mathrm{KOH}$ solution is so low that these planes can be considered as etch stop planes. Precise alignment of the cantilever beams to these planes will, therefore, enable lateral cantilevers to be made with a thickness that only depends on the dimensions of the mask because almost no underetching will occur. An advanced method was used to ensure precise alignment to the crystal planes [11]. The crystal plane etch stop also ensures very smooth surfaces, suitable for interferometric deflection detection.

$\mathrm{KOH}$ etching of the cantilevers is done in two steps: the first step etches the wafer from the backside, defining the width of the cantilevers. In a second step, the cantilevers are etched from the frontside of the wafer. This procedure ensures that the width of the cantilevers can be precisely controlled. The masks used during $\mathrm{KOH}$ etching are thin silicon nitride layers, deposited in a low-stress LPCVD process. SiN layers can withstand the $\mathrm{KOH}$ process, making them a perfect material for fabrication of the tip planes. The SiN masking layer used during $\mathrm{KOH}$ etching is patterned afterwards to form the tip planes. The sharp cutoff corner of the tip plane is obtained by removing the rounded end of the tip plane by means of reactive ion etching using a $\mathrm{Cr}$ mask layer. As a final step, a thin Co coating is evaporated on the front side of the tip plane. To reduce the amount of magnetic material deposited on the sides of the tip plane, accurate alignment of the probe with respect to the evaporation source is needed. A scanning-electron microscope (SEM) photograph of a CantiClever is shown in Fig. 4. The free-hanging tip plane and the smooth sides of the cantilever itself can clearly be distinguished.

\section{MEASUREMENTS}

A CantiClever with a resonance frequency of $60 \mathrm{kHz}$, a 300- $\mu \mathrm{m}$-long cantilever, and a 50-nm Co layer on the 


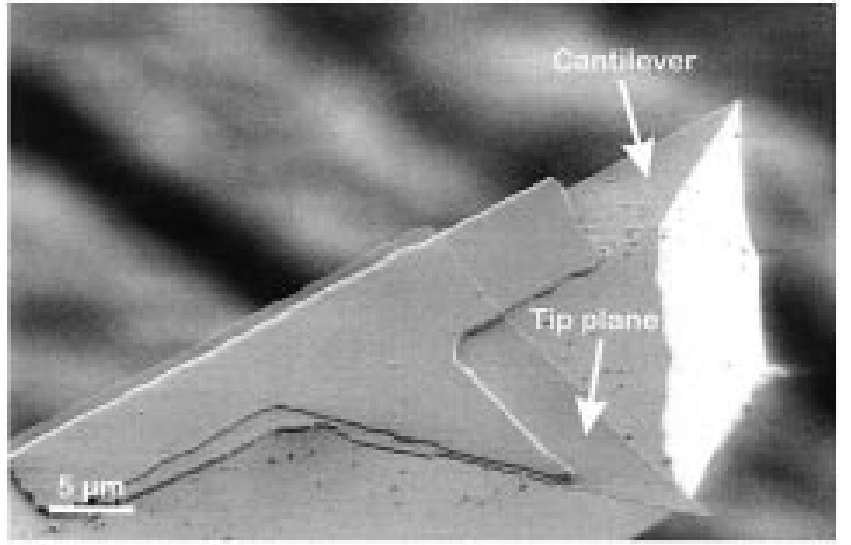

Fig. 4. SEM photograph of the CantiClever showing the cantilever and the tip plane.

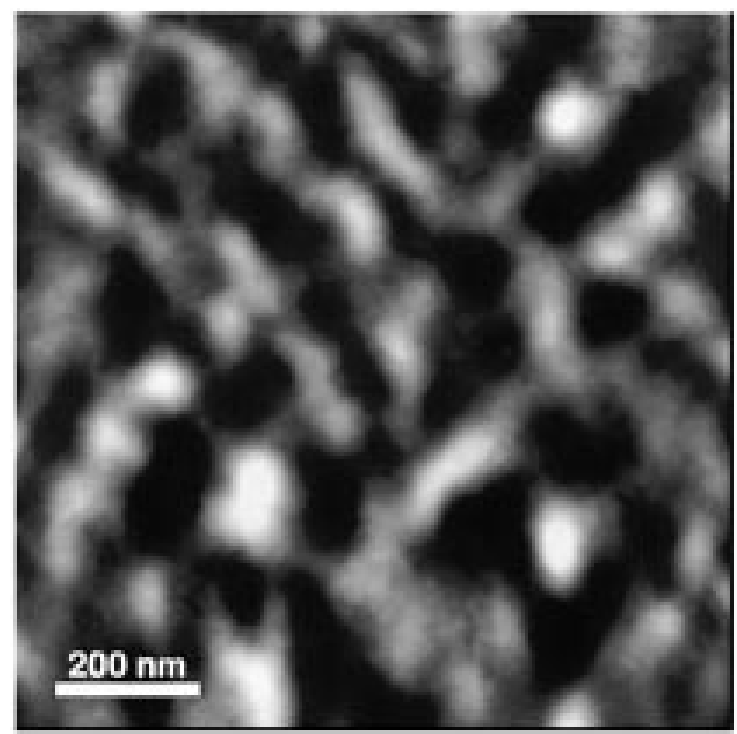

Fig. 5. MFM measurement on a CAMST reference sample.

50-nm-thick tip plane, was mounted in a DI3100 microscope for AFM and MFM measurements.

Fig. 5 shows the result of a $1 \times 1-\mu \mathrm{m}$ MFM scan on a CAMST reference sample as used in [1]. For this scan, the CantiClever was operated in lift mode, detecting the frequency shift at a 15 -nm tip to sample distance. In this scan, magnetic features down to $30 \mathrm{~nm}$ could be distinguished. Currently, work is being done on improvement of the resolution by, among others, reducing the thickness of the tip plane. It is expected that the tip plane thickness can be reduced below $10 \mathrm{~nm}$.

\section{CONCLUSION}

A new design of a cantilever dedicated for MFM has been developed. The new design integrates the cantilever and the tip in one single batch manufacturing process with the use of silicon micromachining techniques. The MFM tip is defined by deposition of a thin magnetic film on the side of a free-standing SiN layer. In this way, both thickness and width of the magnetic tip can be defined very accurately by layer deposition techniques. To facilitate the realization of the free-standing $\mathrm{SiN}$ layer, the cantilever itself is rotated $90^{\circ}$ with respect to conventional manufacturing techniques. A smooth upper surface, suitable for interferometry, is obtained by anisotropic etching of (110) Si wafers in $\mathrm{KOH}$. The manufacturing process is very reliable and a very precise control over the tip diameter is possible. Magnetic features down to $30 \mathrm{~nm}$ could be observed in a CAMST reference sample, using a tip with a $50 \times 50-\mathrm{nm}$ cross section. The authors believe that this technique will allow to produce MFM tips with the ideal tip shape and a cross section below $10 \times 10 \mathrm{~nm}$.

Because the concept of the new probe is centered around the use of a thin film as a support structure one could imagine other types of sensors being integrated on the tip plane. For magnetic imaging, a magnetoresistive element could, for instance, be realized on the tip plane which would make high-resolution magnetoresistance microscopy possible.

\section{REFERENCES}

[1] L. Abelmann, S. Porthun, M. Haast, C. Lodder, A. Moser, M. E. Best, P. J. A. Vanschendel, B. Stiefel, H. J. Hug, G. P. Heydon, A. Farley, S. R. Hoon, T. Pfaffelhuber, R. Proksch, and K. Babcock, "Comparing the resolution of magnetic force microscopes using the CAMST reference samples," J. Magn. Magn. Mat., vol. 190, no. 1-2, pp. 135-147, 1998.

[2] L. Folks, M. E. Best, P. M. Rice, B. D. Terris, D. Weller, and J. N. Chapman, "Perforated tips for high-resolution in-plane magnetic force microscopy," Appl. Phys. Lett., vol. 76, no. 7, pp. 909-911, 2000.

[3] S. Porthun, L. Abelmann, and C. Lodder, "Magnetic force microscopy of thin film media for high density magnetic recording," J. Magn. Magn. Mat., vol. 182, no. 1-2, pp. 238-273, 1998.

[4] S. Porthun, L. Abelmann, S. J. L. Vellekoop, J. C. Lodder, and H. J. Hug, "Optimization of lateral resolution in magnetic force microscopy," Appl. Phys. A-Mater. Sci. Processing, vol. 66, pp. S1185-S1189, 1998.

[5] T. R. Albrecht and C. F. Quate, "Atomic resolution with the atomic force microscope on conductors and non conductors," J. Vac. Sci. Technol. A-Vac. Surf. Films, vol. 6, no. 2, pp. 271-274, 1988.

[6] P. B. Fischer, M. S. Wei, and S. Y. Chou, "Ultrahigh resolution magnetic force microscope tip fabricated using electron beam lithography," J. Vac. Sci. Technol. B, vol. 11, no. 6, pp. 2570-2573, 1993.

[7] G. D. Skidmore and E. D. Dahlberg, "Improved spatial resolution in magnetic force microscopy," Appl. Phys. Lett., vol. 71, no. 22, pp. 3293-3295, 1997.

[8] M. Ruhrig, S. Porthun, J. C. Lodder, S. McVitie, L. J. Heyderman, A. B. Johnston, and J. N. Chapman, "Electron beam fabrication and characterization of high-resolution magnetic force microscopy tips," J. Appl. Phys., vol. 79, no. 6, pp. 2913-2919, 1996.

[9] G. N. Phillips, L. Abelmann, M. Siekman, and J. C. Lodder, "High resolution magnetic force microscopy using focussed ion beam modified tips," Appl. Phys. Lett., 2000, to be published.

[10] L. Meirovitch, Fundamentals of Vibrations, International ed, ser. Mechanical Engineering. New York: McGraw-Hill, 2001.

[11] M. Vangbo and Y. Bäcklund, "Precise mask alignment to the crystallographic orientation of silicon wafers using wet anisotropic etching," $J$. Micromech. Microeng., vol. 6, pp. 279-284, 1996. 